\title{
Monitoring the metabolic side-effects of atypical antipsychotics
}

\author{
Rohit Gumber, ${ }^{1}$ Mizrab Abbas, ${ }^{1}$ Manjunath Minajagi ${ }^{1}$
}

The Psychiatrist (2010), 34, 390-395, doi: 10.1192/pb.bp.109.027433

${ }^{1}$ Leicestershire Partnership NHS Trust, UK

Correspondence to Rohit Gumber (gumber@doctors.org.uk)
Aims and method National clinical guidance states that patients on antipsychotics should have their metabolic profile regularly monitored. The aim of this study was to assess whether we are effectively monitoring metabolic profiles and to improve the detection, communication and intervention of metabolic abnormalities among patients on atypical antipsychotics. We describe a full audit cycle.

Results The audit resulted in a $24 \%$ increase in the number of patients on atypical antipsychotics being referred to the metabolic clinic. The number of abnormal results communicated to primary care showed a significant improvement of $25 \%(P<0.001)$, and ultimately the number of patients who received intervention improved by $17 \%$ $(P=0.001)$.

Clinical implications Audit feedback has been effective in changing clinical practice. The audit demonstrated the potential value of a metabolic clinic and shared care between primary and secondary practitioners for this group of high-risk patients.

Declaration of interest None.
Lifestyle and genetic factors play a part in patients treated with atypical antipsychotics, but metabolic abnormalities occur frequently and their contribution to increased risk is of growing concern to clinicians. ${ }^{1-3}$ Studies indicate that there may be a high risk of metabolic syndrome in patients with schizophrenia independent of medication. ${ }^{4,5}$ However, a larger study found no differences between drug-naive patients with schizophrenia and matched controls, and found that patients receiving antipsychotic drugs experience substantial deposition of both subcutaneous and intra-abdominal fat. ${ }^{6}$ People with metabolic syndrome are three times more likely to suffer a cardiovascular accident or myocardial infarction and have a five times greater risk of developing type 2 diabetes mellitus. ${ }^{3,7}$ The current International Diabetes Federation (IDF) definition of metabolic syndrome is outlined in Table 1.

Various guidelines exist in relation to monitoring the metabolic side-effects of antipsychotics. The National Library for Health produced guidance on the management of adult patients with schizophrenia ${ }^{8}$ based on recommendations in the 2002 National Institute for Health and Clinical Excellence (NICE) schizophrenia guideline. The

Table 1 International Diabetes Federation definition of metabolic syndrome ${ }^{a}$

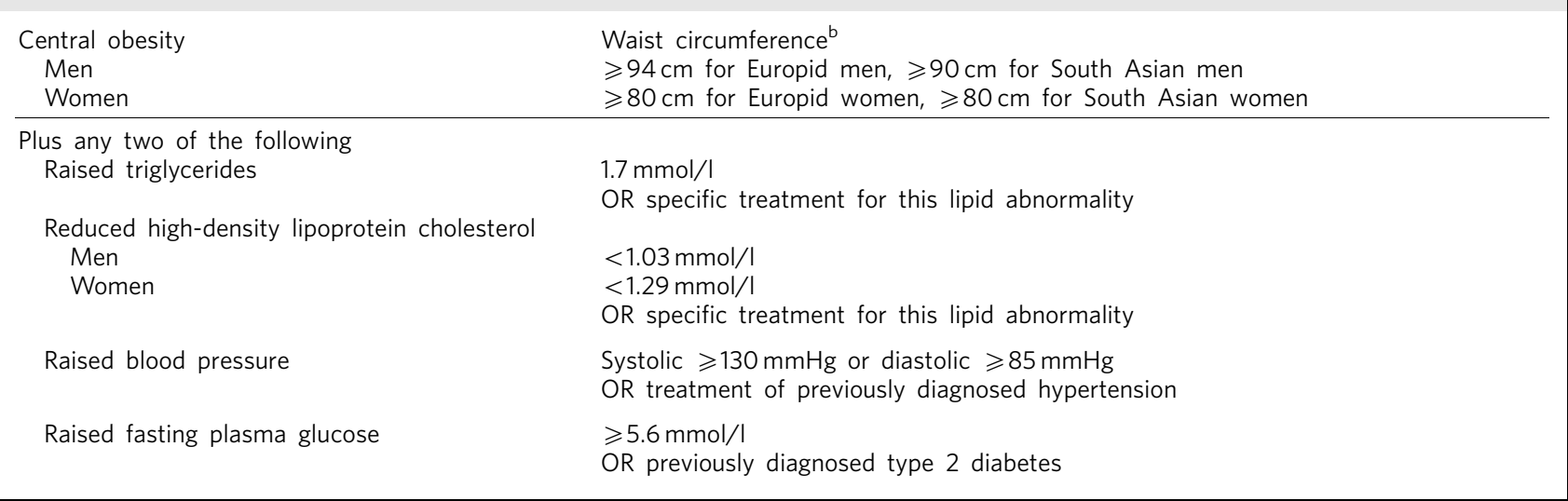

a. Data from International Diabetes Federation, 2006.

b. Other ethnic-specific values exist. 
National Library for Health offers advice concerning the monitoring of adverse effects of antipsychotic drugs, which includes annual monitoring of blood pressure, glucose and calculation of 10-year cardiovascular risk.

The Maudsley Prescribing Guidelines ${ }^{9}$ provide guidance on monitoring parameters of patients on antipsychotics. They recommend the following:

- fasting blood lipids (cholesterol, triglycerides) at baseline, 3 months, then yearly (clozapine, olanzapine, quetiapine: 3-monthly for first year, then yearly);

- weight (including waist size and body mass index (BMI)) at baseline, frequently for 3 months then yearly (clozapine, olanzapine, quetiapine: frequently for 3 months, then 3-monthly for first year, then yearly);

- plasma glucose at baseline, at 4-6 months, then yearly (clozapine, olanzapine: at baseline, 1 month and then every 4-6 months);

- blood pressure at baseline and frequently during dose titration.

Barnett et $a l^{3}$ recommend baseline evaluation of cardiovascular risk factors followed by 4-, 8- and 12-week evaluations and a 6-month evaluation, and annual monitoring thereafter. In addition, they recommend that all patients with weight gain should receive lifestyle advice in combination with close monitoring, and patients with hyperglycaemia, dyslipidaemia and hypertension should be referred to their general practitioner (GP) for appropriate treatment according to current guidelines.

The national Prescribing Observatory for Mental Health (POMH-UK) has included screening for the metabolic syndrome in community patients receiving antipsychotics as a topic for its quality improvement programme. The group conducted a retrospective casenote audit of patients prescribed antipsychotic medication with a standard of yearly monitoring of blood pressure, measure of obesity, glucose and lipids. Results showed that between 0 and $41 \%$ ( 0 and $48 \%$ at re-audit a year later) of trusts were monitoring for all four aspects on an annual basis. The group suggested that there was considerable unrecognised diabetes, hypertension and dyslipidaemia within this patient group. The responses of clinical teams showed that there were obstacles to screening, revealing uncertainty about whose responsibility it was and a lack of confidence in interpretation of abnormal results. ${ }^{2,10}$ Haupt et $a l^{11}$ sought to quantify plasma lipid and glucose testing rates in patients receiving second-generation antipsychotics before and after guidelines recommending testing were issued. Despite a significant improvement after guidelines were issued, the authors conclude that monitoring for plasma lipids and glucose remains low.

The Charnwood mental health team, Leicestershire Partnership National Health Service (NHS) Trust, started a metabolic clinic in 2006 to monitor for metabolic sideeffects in patients on regular antipsychotic medication. The Charnwood area is in the East Midlands region of the UK and consists of both urban and rural areas. The population is about 155000 with $11 \%$ being ethnic minority (mainly Indian and Other White). Patients are referred by psychiatrists in the Charnwood team. The clinic is run by junior specialty trainees and involves monitoring of metabolic parameters at baseline, 3 months, 6 months and at 1 year. Abnormal results are sent to GPs for their attention and any required intervention. At the end of 1 year, the responsibility of annual monitoring is then passed on to GPs. Specialty trainees are trained by a consultant psychiatrist in an induction session and are supervised in the initial sessions. A clinic file also provides an outline of how the clinic is run.

The aims of this study were to assess whether we are effectively monitoring the patients' metabolic profile, and to improve the detection (number of patients referred to clinic and number of results completed in clinic), communication (number of results received by GPs) and intervention (number of results acted on) of metabolic abnormalities among patients on atypical antipsychotics.

\section{Method}

The study was based on the results from the metabolic clinic that are recorded on a standard form (pro forma) at each visit. The initial audit was carried out on data between May 2006 and December 2007, involving 69 patients on the clinic register. The re-audit was carried out on data between December 2007 and January 2009, involving 123 patients on the clinic register.

The standards for the audit were set after considering our local 'shared care agreement' between secondary and primary care, national ${ }^{9}$ and international ${ }^{7}$ guidelines, and after consultation with a consultant biochemist at the University Hospitals of Leicester NHS Trust. The shared care agreement recommends monitoring at baseline, 4 months and 12 months; however, based on national and international guidelines, we felt that baseline, 3-month, 6 -month and 12-month monitoring of all parameters would be more acceptable and practical. From these we devised the following standards.

1. All patients on atypical antipsychotics should be referred to the metabolic clinic.

2. All patients should have their BMI, waist circumference, blood pressure, lipid profile and fasting or random glucose tested at baseline and at 3, 6 and 12 months.

3. Results are abnormal and should be sent to GPs and acted on if:

(i) $\mathrm{BMI} \geqslant 30 \mathrm{~kg} / \mathrm{m}^{2}$

(ii) waist circumference $\geqslant 94 \mathrm{~cm}$ for males and $\geqslant 80 \mathrm{~cm}$ for females

(iii) total cholesterol $\geqslant 4.6 \mathrm{mmol} / \mathrm{l}$

(iv) triglycerides $\geqslant 1.7 \mathrm{mmol} / \mathrm{l}$

(v) high-density lipoprotein cholesterol $<1.03 \mathrm{mmol} / \mathrm{l}$ for males and $<1.29 \mathrm{mmol} / \mathrm{l}$ for females

(vi) systolic blood pressure $\geqslant 130 \mathrm{mmHg}$

(vii) diastolic blood pressure $\geqslant 85 \mathrm{mmHg}$

(viii) fasting plasma glucose $\geqslant 5.6 \mathrm{mmol} / \mathrm{l}$

(ix) random plasma glucose $\geqslant 6.6 \mathrm{mmol} / \mathrm{l}$.

To test the first standard, a random sample of patients on atypical antipsychotics was required. A list of all patients on atypical antipsychotics in the Charnwood mental health team was generated. Ten patients from each of the four consultants on the team were selected using systemic 
randomisation (e.g. if a consultant has 64 patients on atypical antipsychotics then every 6th patient was selected to total ten). This list was then compared with the list of patients that have had metabolic monitoring at some time in the metabolic clinic and the proportion of patients referred was calculated.

Attendances and clinic results at baseline, 3 months, 6 months and yearly were retrospectively reviewed and analysed. Patients on typical antipsychotics, patients who had moved to other mental health teams and those not on regular antipsychotics were excluded. The number of parameters completed and the number of abnormal results were calculated.

To test whether the abnormal results had been acted on, only the patients' last clinic results were used. We contacted the GPs by telephone and fax to ascertain whether they had received their patients' abnormal results and whether they had acted on them (e.g. by providing lifestyle advice, prescribing medication, making a relevant referral). They were also given the opportunity to make comments. We thought this to be the most feasible way of obtaining relevant information as it would be unreasonable to ask GPs to go through notes of patients to check multiple clinic dates, sometimes as far back as 2006.

Statistical analysis to compare data in both audits was carried out using contingency tables. Fisher's exact test (two-tailed) was used, with a 5\% significance level.

\section{Results}

The study group of the initial audit comprised 54 patients (after exclusion criteria), with a male:female ratio of 29:25 and an average age of 40.7 years. The proportion of patients referred to the metabolic clinic was $43 \%$. Of the 48 patients that attended baseline appointments, $35 \%$ had a diagnosis of metabolic syndrome as defined by the IDF. It was shown that $63 \%$ of abnormal results were received by GPs and $66 \%$ of received results were acted on. General practitioners received a higher number of haematological results than non-haematological results. Furthermore, the number of incomplete high-density lipoprotein results was relatively high at $21 \%$.
The audit was presented to trainees and consultants in January 2008; the following recommendations were implemented in December 2007.

- Metabolic clinic file to include an outline of the aims of the clinic and how it is run.

- Pro formas to include IDF criteria and normal/ abnormal parameters; a checklist of investigations should be completed; a space to be incorporated to record whether pro formas (not just blood results) are faxed to the GP, and to note lifestyle advice or changes to medication and action on previous results.

- High-density lipoprotein should be specifically requested on chemical pathology forms.

- Specialty trainees to receive education on metabolic syndrome.

- General practitioners to be informed of antipsychotic monitoring guidelines and the effects of antipsychotics on metabolic profile.

The audit was repeated with the study group comprising 110 patients, with a male:female ratio of $63: 47$ and an average age of 38.2 years.

Analysis of all clinic results (number of sets of clinic results: initial audit, $n=96$; re-audit, $n=184$ ) revealed a large number of abnormal results (defined by audit standards). In both audits, waist circumference and total cholesterol had the highest number of abnormal results (Table 2).

\section{Detection of abnormal results}

The proportion of patients referred to the clinic at re-audit was $67 \%$, an increase of $24 \%$ on the initial audit. Highdensity lipoprotein, which was not completed in $21 \%$ of cases in the initial audit, had improved in the re-audit to $16 \%$ not being completed. However, the number of glucose results not completed increased from $8 \%$ to $18 \%$ in the re-audit.

The response from GPs to our request for information on results received and acted on was high (over 85\%); however, 25 sets of abnormal results from the initial audit and 23 from the re-audit did not receive a response from GPs.

\begin{tabular}{|c|c|c|c|c|c|c|c|c|c|c|}
\hline \multirow[b]{2}{*}{ Parameter } & \multicolumn{2}{|c|}{ Normal } & \multicolumn{2}{|c|}{ Abnormal } & \multicolumn{2}{|c|}{ Not done } & \multicolumn{2}{|c|}{ Refused } & \multicolumn{2}{|c|}{ Results pending } \\
\hline & A & B & A & B & A & B & A & B & A & B \\
\hline Body mass index & 44 & 49 & 55 & 51 & 1 & 0 & 0 & 0 & 0 & 0 \\
\hline Waist circumference & 13 & 13 & 86 & 87 & 1 & 0 & 0 & 0 & 0 & 0 \\
\hline Systolic blood pressure & 64 & 59 & 36 & 40 & 0 & 1 & 0 & 0 & 0 & 0 \\
\hline Diastolic blood pressure & 74 & 72 & 26 & 27 & 0 & 1 & 0 & 0 & 0 & 0 \\
\hline Total cholesterol & 25 & 20 & 69 & 73 & 1 & 3 & 4 & 3 & 1 & 1 \\
\hline Triglycerides & 46 & 40 & 48 & 51 & 1 & 5 & 4 & 3 & 1 & 1 \\
\hline High-density lipoprotein & 34 & 45 & 40 & 35 & 21 & 16 & 4 & 3 & 1 & 1 \\
\hline Plasma glucose fasting or random ${ }^{b}$ & 69 & 58 & 10 & 14 & 8 & 18 & 3 & 3 & 3 & 1 \\
\hline
\end{tabular}

A, initial audit; B, re-audit

a. Initial audit $n=96$, re-audit $n=184$

b. $7 \%$ of patients known to have diabetes at initial audit and $6 \%$ at re-audit. 


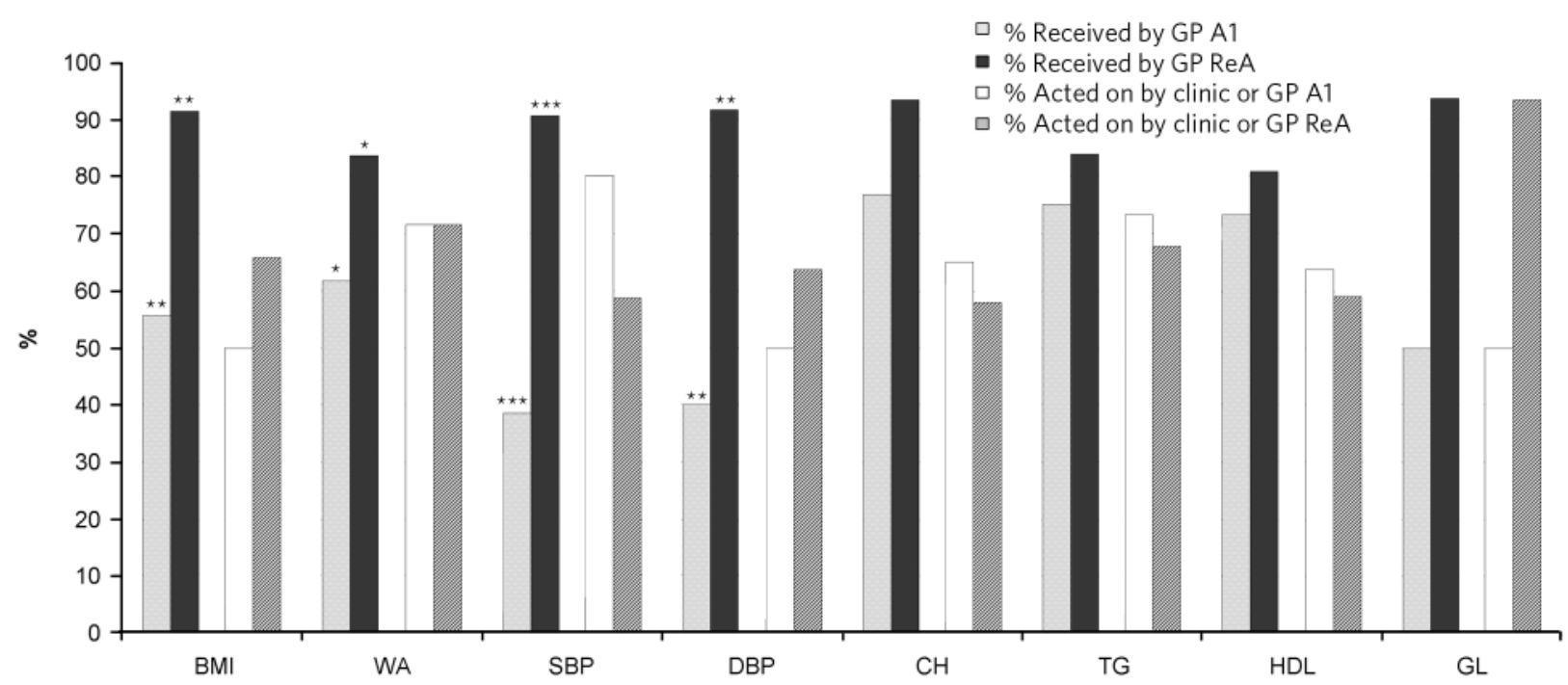

Fig 1 Abnormal results received by the general practitioners and received results acted on (excluding results unknown), with ${ }^{\star} P<0.05$, ${ }^{\star} P<0.01$, ${ }^{\star \star \star} P<0.001$ for differences between audits for each parameter.

A1, audit; ReA, re-audit; BMI, body mass index; $\mathrm{CH}$, cholesterol; DBP, diastolic blood pressure; GL, glucose; GP, general practitioner; HDL, high-density lipoprotein; SBP, systolic blood pressure; TG, triglycerides; WA, waist circumference.

\section{Communication of abnormal results}

In the re-audit, GPs received on average $88 \%$ of abnormal results, an increase of $25 \%$ on the initial audit $(P<0.001)$.

Significant improvements were seen between audits for the number of abnormal results received by GPs for systolic blood pressure, diastolic blood pressure, BMI and waist circumference $(52 \%, 52 \%, 36 \%$ and $22 \%$ improvement respectively). The improvement in number of abnormal cholesterol results received (17\%) was of borderline significance $(P=0.06)$. The number of abnormal glucose results received did not significantly improve $(P=0.09)$ because of the low number of results for the initial audit; however, Fig. 1 indicates a noticeable improvement. The number of abnormal triglycerides and high-density lipoprotein results received by GPs showed no significant improvement (probably due to initial audit results being relatively high).

\section{Action on abnormal results}

Number of results acted on by GPs showed no significant change (on average, $66 \%$ in both audits). Some, but not all, GPs provided reasons for not acting in the comments section: many believed the 'abnormal' result was not abnormal enough to warrant further action after weighing up the risks for that individual patient.

\section{Overall communication of and action on abnormal results}

The overall number of abnormal results received and acted on improved to some extent for all eight parameters. Notably, overall action on abnormal glucose results improved by $63 \%(P=0.03)$, BMI by $32 \%(P=0.04)$ and diastolic blood pressure by $38 \%(P=0.06)$. The total number of abnormal results received and acted on increased from
$41 \%$ to $58 \%$, an increase of $17 \%(P=0.001)$ on the initial audit.

\section{Discussion}

In this audit cycle we were able to produce a notable improvement in monitoring and communication of abnormal results to primary care services. We found that a large proportion of patients taking atypical antipsychotics have abnormal metabolic parameters; this underlines the need for monitoring all patients taking these medications regularly. At re-audit, of those who had a measurement, $87 \%$ had an abnormal waist circumference, $78 \%$ abnormal cholesterol and 19\% abnormal glucose (excluding those known to have diabetes).

In the re-audit, consultants' referral rates were improved at $67 \%$, albeit still well below the audit standard. This reflects the limited capacity of the metabolic clinic and it is possible that only high-risk patients are being referred. There may also have been a number of patients who refused to attend the clinic. Consequently, the high numbers of abnormal parameters may be skewed by referral bias. The results may also have been affected by non-attendance meaning incomplete follow-up of these patients.

Despite implementation of audit recommendations, high-density lipoprotein and glucose tests were insufficiently requested by specialty trainees and the number of glucose results not completed in fact increased. Although the reason behind this is unclear, this does indicate the need for further education of specialty trainees and clarity on blood test forms. In the trust, blood for glucose testing is requested in a separate bottle, which may be a reason it is getting omitted.

Overall, $12 \%$ of abnormal results were not sent to the GP; some were not returned to the correct department by the laboratory but it is likely that trainees failed to recognise others as abnormal. Despite this, there was an 
improvement in the number of abnormal results being sent to GPs and acted on between the two audits, notably for glucose, BMI and diastolic blood pressure. The most significant of these is the increase of $63 \%$ for abnormal glucose results, which probably reflects the successful implementation of recommendations from the initial audit, especially the provision of IDF criteria and education on metabolic syndrome.

Communication of abnormal blood results between the clinic and primary care improved by $25 \%$ between audits (especially for the non-haematological tests), leading to an overall increased action on abnormal results of $17 \%$. On average, GPs acted on $66 \%$ of abnormal results in both audits. Many believed the results were 'not abnormal enough' to warrant further action. There is a case that all abnormal results should be acted on as most patients only require low-key intervention such as lifestyle advice and this may prevent patients developing metabolic syndrome. Conversely, it is true that all patients should be looked at individually and risks assessed before action. Some GPs believed the clinic already offered sufficient lifestyle advice. This is true; however, the advice is only based on parameters completed at the time of clinic (blood pressure, waist circumference and BMI) and not blood results (cholesterol, triglycerides, high-density lipoprotein and glucose). This means many patients would not receive intervention.

Our results show that we are now monitoring $67 \%$ of patients on atypical antipsychotics in secondary care. Of these, the vast majority (over 95\%) have waist circumference, BMI, blood pressure, cholesterol and triglycerides assessed regularly, but high-density lipoprotein and glucose are tested in fewer patients ( $84 \%$ and $82 \%$ respectively). The results compare favourably with the audit conducted by Barnes et $a l,{ }^{10}$ which found even at re-audit that a documented annual result for blood pressure was available for $43 \%$ of patients, BMI or other obesity measure for $34 \%$, blood glucose for $38 \%$ and lipids for $35 \%$; only $23 \%$ of patients had all four measures completed. Although the audit does report numbers of patients with diabetes, dyslipidaemia and hypertension, they did not examine specific results abnormalities or any intervention due to these abnormalities. Our results also compare favourably with Haupt et al, ${ }^{11}$ who reported comparatively low rates of glucose and lipid testing. However, the improvement in monitoring which we have observed reflects that in Haupt et $a l$ 's study, where a significant improvement on testing rates post-guidance was reported. Our audit goes further than reporting solely the levels of monitoring; it provides information on specific numbers of abnormal results and interventions involving both secondary and primary care.

\section{Implications for clinical practice}

The responsibility for monitoring is an unclear area, as demonstrated by Barnes et $a l^{10}{ }^{10}$ where teams expressed uncertainty about responsibility for physical health screening. The NICE guidelines for schizophrenia ${ }^{12}$ state that physical health should be monitored and recorded 'regularly and systematically throughout treatment, but especially during titration' and that 'GPs and other primary healthcare professionals should monitor the physical health of people with schizophrenia at least once a year'. It also states that 'Healthcare professionals in secondary care should ensure, as part of the care programme approach, that people with schizophrenia receive physical healthcare from primary care'.

A shared care agreement is a possible way to enhance care of patients on atypical antipsychotics. This agreement addresses responsibilities for prescribing between the specialist and general practitioner. The audit shows that this way of working can be successful as we have demonstrated higher rates of monitoring than previous studies involving multiple trusts. It is essential that all information is provided to the GP. Education and training of specialty trainees on the metabolic syndrome and what constitutes an abnormal result contributed to a $25 \%$ increase in communication of abnormal results to primary care.

The Charnwood metabolic clinic has made changes to the way it is run as a result of audit findings. A Healthy Living Group has been set up as part of intervention in addition to GP services. The aims are to give lifestyle advice and supervised exercise programmes to patients who attend the clinic and are diagnosed with metabolic syndrome. Referrals are made via the specialty trainee in the clinic. Patients are now given four appointments at the beginning of their 1-year monitoring and reminders are sent at each interval. On completion of 1-year monitoring the final pro forma with all results is faxed to the GPs, who are requested to continue the monitoring annually thereafter. The clinic is currently run by junior specialty trainees. In order to meet the audit standards, they would require further support, possibly from nursing staff trained in phlebotomy. This would leave time in the clinic for other tasks such as informing GPs and consultants of abnormal results. The role of the doctor could eventually be completely taken over by nursing staff.

The metabolic profile of patients on atypical antipsychotics is clearly of concern. Psychiatrists and GPs must work together to carry out efficient monitoring of all relevant parameters so that early interventions to reduce the risk of cardiovascular disease and type 2 diabetes are possible.

\section{Acknowledgements}

We would like to thank the Charnwood mental health team, the administration staff and all the GPs in the clinic catchment area for their help and support.

\section{About the authors}

Rohit Gumber and Mizrab Abbas are specialty trainees in psychiatry and Manjunath Minajagi is consultant in general adult psychiatry in the Bradgate Mental Health Unit, Glenfield General Hospital, Leicester, Leicestershire Partnership NHS Trust.

\section{References}

1 De Hert $M$, Hanssens $L$, Van Winkel $R$, Wampers $M$, Van Eyck $D$ Scheen $A$, et al. A case series: evaluation of the metabolic safety of aripiprazole. Schizophr Bull 2007; 33: 823-30. 
2 Prescribing Observatory for Mental Health (POMH-UK). The Metabolic Side Effects of Antipsychotic Medication Represent a Serious Risk to Physical Health. POMH-UK, 2006 (http://www.rcpsych.ac.uk/pdf/ T2\%20info\%20leaflet.pdf)

3 Barnett $\mathrm{AH}$, Mackin P, Chaudhry I, Faroogi A, Gadsby R, Heald A, et al. Minimising metabolic and cardiovascular risk in schizophrenia: diabetes, obesity and dyslipidaemia. J Psychopharmacol 2007; 21: 357-73.

4 Thakore JH, Mann JN, Vlahos I, Martin A, Reznek R. Increased visceral fat distribution in drug-naive and drug-free patients with schizophrenia. Int J Obesity 2002; 26: 137-41.

5 Ryan MC, Flanagan S, Kinsella U, Keeling F, Thakore JH. The effects of atypical antipsychotics on visceral fat distribution in first episode, drugnaive patients with schizophrenia. Life Sci 2004; 74: 1999-2008.

6 Zhang ZJ, Yao ZJ, Liu W, Fang Q, Reynolds GP. Effects of antipsychotics on fat deposition and changes in leptin and insulin levels. Magnetic resonance imaging study of previously untreated people with schizophrenia. Br J Psychiatry 2004; 184: 58-62.
7 International Diabetes Federation (IDF). The IDF Consensus Worldwide Definition of Metabolic Syndrome. IDF, 2006 (http://www.idf.org/ webdata/docs/IDF_Meta_def_final.pdf).

8 National Library for Health. Clinical Knowledge Summaries: Schizophrenia. National Library for Health, 2007 (http://www.cks.library.nhs.uk/ schizophrenia).

9 Taylor D, Paton C, Kerwin R. Antipsychotics - monitoring In The Maudsley Prescribing Guidelines (9th edn). Informa Healthcare, 2007.

10 Barnes TRE, Paton C, Cavanagh MR, Hanock E, Taylor DM. A UK audit of screening for the metabolic side effects of antipsychotics in community patients. Schizophr Bull 2007; 33: 1397-1403.

11 Haupt DW, Rosenblatt LC, Kim E, Baker RA, Whitehead R, Newcomer JW. Prevalence and predictors of lipid and glucose monitoring in commercially insured patients treated with second-generation antipsychotic agents. Am J Psychiatry 2009; 166: 345-53.

12 National Institute for Health and Clinical Excellence. Schizophrenia: Core Interventions in the Treatment and Management of Schizophrenia in Adults in Primary and Secondary Care (CG82). NICE, 2009. 